\title{
INVERSION AND SUBSPACES OF A FINITE FIELD
}

\author{
SANDRO MATTAREI
}

\begin{abstract}
Consider two $\mathbb{F}_{q}$-subspaces $A$ and $B$ of a finite field, of the same size, and let $A^{-1}$ denote the set of inverses of the nonzero elements of $A$. The author proved that $A^{-1}$ can only be contained in $A$ if either $A$ is a subfield, or $A$ is the set of trace zero elements in a quadratic extension of a field. Csajbók refined this to the following quantitative statement: if $A^{-1} \nsubseteq B$, then the bound $\left|A^{-1} \cap B\right| \leq 2|B| / q-2$ holds. He also gave examples showing that his bound is sharp for $|B| \leq q^{3}$. Our main result is a proof of the stronger bound $\left|A^{-1} \cap B\right| \leq|B| / q \cdot\left(1+O_{d}\left(q^{-1 / 2}\right)\right)$, for $|B|=q^{d}$ with $d>3$. We also classify all examples with $|B| \leq q^{3}$ which attain equality or near-equality in Csajbók's bound.
\end{abstract}

\section{INTRODUCTION}

In response to a question of Andrea Caranti, for use in [CDVS09], the author determined in [Mat07] the additive subgroups of a field which are closed with respect to inverting nonzero elements. The more general question with a division ring instead of a field was independently answered in [GGSZ06]. The proofs depend on Hua's identity [Hua49], and on Jordan algebra techniques to cover the noncommutative case. However, a more direct argument based on polynomials was given in [Mat07] in the special case of finite fields, which appears to have attracted some attention for cryptographic applications. In that special case the result reads as follows: a non-trivial inverse-closed additive subgroup $A$ of a finite field $E$ is either a subfield of $E$ or the set of elements of trace zero in some quadratic field extension contained in $E$.

In [Csa13], Bence Csajbók investigated a question which may be thought of as a refinement of this result: can one obtain the same conclusion from the weaker assumption that $A$ is an additive subgroup of a finite field which is almost inverseclosed, in the sense that most of the inverses of its nonzero elements belong to $A$ ? Of course the two words in italics need to be given a precise meaning. A very special case of this occurred in [KLS12, Lemma 5.3], where the conclusion was proved under the assumption that $A$ is inverse closed up to at most two nonzero elements.

It turns out that this question is better studied in the more general form where two additive subgroups $A$ and $B$ of the same size of a finite field are considered, and one asks for an upper bound on $\left|A^{-1} \cap B\right|$ in terms of $|B|$ in case $A^{-1} \nsubseteq B$.

Date: February 21, 2014.

2000 Mathematics Subject Classification. Primary 11T06; secondary 51E20.

Key words and phrases. finite field, subspace, inversion. 
Here $S^{-1}$, for $S$ a subset of a field, denotes the set of inverses of the nonzero elements of $S$. Note that the intersection $A^{-1} \cap B$ attains maximal size $q^{d}-1$ exactly when $A^{-1} \subseteq B$, and in that case $A$ and $B$ are both (one-dimensional)

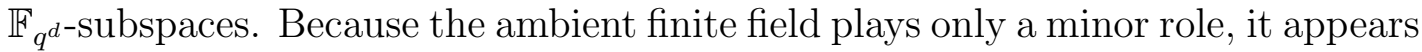
convenient to work in the algebraic closure of a finite field, and so we rather state Csajbók's results in the following equivalent form.

Theorem 1 (Theorems 1.2 and 3.1 in [Csa13]). Let $A$ and $B$ be finite nonzero $\mathbb{F}_{q^{-}}$subspaces of $\overline{\mathbb{F}_{q}}$ of the same size, with $A^{-1} \nsubseteq B$. Then $\left|A^{-1} \cap B\right| \leq 2|B| / q-2$.

When $q=2$ and $|B|>2$ the conclusion can be strengthened to $\left|A^{-1} \cap B\right| \leq$ $3|B| / 4-1$.

In Section 2 we present a proof of Theorem 1 which is shorter than Csajbók's original proof, but also more explicit. This is because, say in case of the former bound of Theorem 1, our proof produces a polynomial $C(x)$, of degree $2|B| / q-2$, explicitly computable from the polynomials defining $A$ and $B$, whose set of roots contains $A^{-1} \cap B$. This can then be effectively used for further study of $A^{-1} \cap B$, as we illustrate next.

A natural question which arises at this point is whether the bounds given in Theorem 1 are best possible, especially the general bound which holds for arbitrary $q$. In the early draft of [Csa13] which was available to the author during most of the writing of this paper, the few examples provided fell short of showing sharpness of the bound beyond the rather trivial cases where $|B| \leq q^{2}$, which we briefly discuss at the end of Section 2 .

The present work begun as an attempt to provide examples with $|B|=q^{3}$ where equality is attained in Csajbók's bound. We present such examples in Section 3. They appear in Theorem 4, within a more general situation where $\left(A^{-1} \cap B\right) \cup\{0\}$ contains a one-dimensional $\mathbb{F}_{q^{2}}$-subspace of $\overline{\mathbb{F}_{q}}$. In fact, under this assumption, which we will later show not to be restrictive, our proof of Theorem 1 is especially effective: it allows us to give a polynomial description of all pairs $(A, B)$ of three-dimensional spaces which attain equality in Csajbók's bound, that is, which satisfy $\left|A^{-1} \cap B\right|=2 q^{2}-2$. This is possible only for $q$ odd and, in geometric language, it occurs exactly when the image of $A^{-1} \cap B$ in the projective plane $\mathbb{P} B \cong \mathbb{P}^{2}\left(\mathbb{F}_{q}\right)$ associated with the linear $\mathbb{F}_{q}$-space $B$ is the union of a (nondegenerate) conic and an external line. The configuration of the union of a conic and a secant line also arises, and the corresponding subspaces then satisfy $\left|A^{-1} \cap B\right|=2 q^{2}-2 q$. In even characteristic those two configurations collapse to that of a conic and a tangent line, whence $\left|A^{-1} \cap B\right|=2 q^{2}-q-1$. The final, published version of [Csa13], includes a study of the case where $A$ and $B$ have dimension three, providing a presentation of the examples which we have briefly described. However, Csajbók's geometric approach limits his results to subspaces of $\mathbb{F}_{q^{4}}$ for $q$ odd, and there is little overlap with our results, see Remark 8.

The claim we implicitly made above, that we have actually found all pairs $(A, B)$ of three-dimensional $\mathbb{F}_{q}$-subspaces of $\overline{\mathbb{F}_{q}}$ which attain equality in Csajbók's bound, relies on removing our additional assumption that $\left(A^{-1} \cap B\right) \cup\{0\}$ contains 


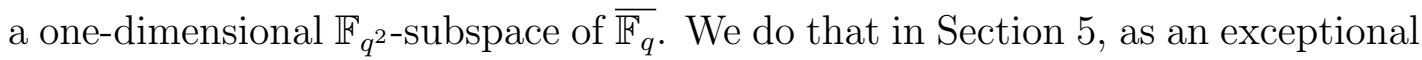
case of a more general goal which we now introduce.

Csajbók speculated in [Csa13] that for $\mathbb{F}_{q}$-subspaces $A$ and $B$ of the same fixed dimension $d>3$ the stronger bound $\left|A^{-1} \cap B\right| \leq|B| / q \cdot\left(1+O\left(q^{-1 / 2}\right)\right)$ might hold. Our main result shows that this is indeed the case.

Theorem 2. Let $A$ and $B$ be finite nonzero $\mathbb{F}_{q}$-subspaces of $\overline{\mathbb{F}_{q}}$, with $|A|=|B|=$ $q^{d}>q^{3}$ and $A^{-1} \nsubseteq B$. Then

$$
\left|A^{-1} \cap B\right| \leq q^{d-1}+(d-1)(d-2) q^{d-(3 / 2)}+C_{d} \cdot q^{d-2},
$$

where $C_{d}$ only depends on $d$.

It follows, in particular, that Csajbók's general bound of Theorem 1 can only be sharp for subspaces of dimension up to three (at least for large $q$ ).

Corollary 3. Equality in the bound $\left|A^{-1} \cap B\right| \leq 2|B| / q-2$ of Theorem 1 is never attained for $d>3$, where $|B|=q^{d}$, provided $q$ is sufficiently large with respect to $d$.

The bound of Theorem 2, which we prove in Section 4, results from an application of the Lang-Weil bound to a multivariate polynomial closely related to the polynomial $C(x)$ used in our proof of Theorem 1, when that is irreducible. However, considerable work is necessary, which we postpone to Section 6 , to show that such polynomial can only be reducible in very special situations. Those exceptional geometric situations generalise the configurations of pairs of twoor three-dimensional subspaces attaining equality in Csajbók's bound which we have briefly discussed earlier.

\section{Intersecting a SUbSPaCe With the INVERSE OF ANOTHER}

A finite subset of $\overline{\mathbb{F}_{q}}$ is conveniently characterised by the unique monic polynomial in $\overline{\mathbb{F}_{q}}[x]$ whose roots are the elements of the subset, each with multiplicity one. Thus, to the $\mathbb{F}_{q}$-subspaces $A$ and $B$ of $\overline{\mathbb{F}_{q}}$, with size $q^{d}$, throughout the paper we associate the monic polynomials which have the elements of $A$ and $B$, respectively, as their roots, each with multiplicity one. (Using the same letters for the subspaces $A$ and $B$ and their polynomials should create no confusion.) It is well known that $A(x)$ and $B(x)$ are $q$-polynomials, see [LN83, Theorem 3.52], which means that they have the form $A(x)=\sum_{i=0}^{d} a_{i} x^{q^{i}}$ and $B(x)=\sum_{i=0}^{d} b_{i} x^{q^{i}}$, with $a_{d}=b_{d}=1$. Also, the simplicity of their roots amounts to $a_{0} b_{0} \neq 0$. Hence the roots of $x^{q^{d}} A(1 / x)=\sum_{i=0}^{d} a_{i} x^{q^{d}-q^{i}}$ and $B(x) / x$ are the elements of $A^{-1}$ and $B \backslash\{0\}$, respectively. With this notation at hand we now present a very short proof of Csajbók's bounds.

Proof of Theorem 1. The idea of the proof is to give an upper bound on the degree of the greatest common divisor of $x^{q^{d}} A(1 / x)$ and $B(x) / x$. The fact that the non-leading terms of $B(x)$ have relatively small degree suggests applying a variant of polynomial long division, where one keeps subtracting a scalar multiple 
of $B(x) / x$ from the current reminder multiplied by the appropriate power of $x$. The final result of this process is condensed in the following argument.

The common roots of $x^{q^{d}} A(1 / x)$ and $B(x) / x$ are also roots of the polynomial

$$
\begin{aligned}
C(x) & =x^{q^{d}} A(1 / x) \cdot x^{q^{d-1}-1}-B(x) / x \cdot x^{q^{d-1}}\left(A(1 / x)-1 / x^{q^{d}}\right) \\
& =x^{q^{d-1}-1}-\left(B(x)-x^{q^{d}}\right) / x \cdot x^{q^{d-1}}\left(A(1 / x)-1 / x^{q^{d}}\right),
\end{aligned}
$$

which has degree at most $2 q^{d-1}-2$. This shows that $\left|A^{-1} \cap B\right| \leq 2|B| / q-2$, except when the polynomial $C(x)$ vanishes. The latter condition occurs exactly when $A(x)=x^{q^{d}}+a_{0} x$ and $B(x)=x^{q^{d}}+a_{0}^{-1} x$, which means that $A$ and $B$ are

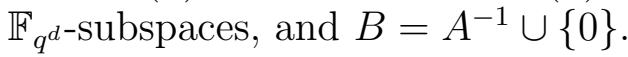

Assuming $d>1$ we now prove a different bound, which holds for arbitrary $q$ but improves on the previous bound only when $q=2$. The polynomial

$$
\begin{aligned}
D(x) & =C(x) \cdot x^{q^{d}-2 q^{d-1}+1}+b_{d-1} x^{q^{d}} A(1 / x) \\
& =x^{q^{d}-q^{d-1}}+b_{d-1}-\left(B(x)-x^{q^{d}}-b_{d-1} x^{q^{d-1}}\right) \cdot x^{q^{d-q^{d-1}}}\left(A(1 / x)-1 / x^{q^{d}}\right),
\end{aligned}
$$

has degree at most $q^{d}-q^{d-1}+q^{d-2}-1$, and is nonzero if $A$ and $B$ are not both

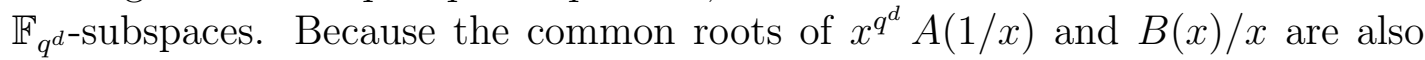
roots of $D(x)$ we obtain $\left|A^{-1} \cap B\right| \leq q^{d}-q^{d-1}+q^{d-2}-1$. This is better than the previous bound only when $q=2$, and then reads $\left|A^{-1} \cap B\right| \leq 3|B| / 4-1$.

The above proof offers more advantages over Csajbók's original proof than just brevity. It provides us with a polynomial

$$
C(x)=x^{q^{d-1}-1}-\left(\sum_{i=0}^{d-1} a_{i} x^{q^{d-1}-q^{i}}\right) \cdot\left(\sum_{j=0}^{d-1} b_{j} x^{q^{j}-1}\right),
$$

of degree at most $2 q^{d-1}-2$, such that all elements of $A^{-1} \cap B$ are roots of $C(x)$. We will put that to good use in the next sections.

We mention in passing that our proof of Theorem 1 can be easily modified to deal with affine $d$-dimensional $\mathbb{F}_{q}$-subspaces of $\overline{\mathbb{F}_{q}}$. In fact, such affine subspaces have the form $A+\alpha$ and $B+\beta$, with $A, B$ as in Theorem 1 and $\alpha, \beta \in \overline{\mathbb{F}_{q}}$, which are the sets of roots of the polynomials $A(x-\alpha)=A(x)-A(\alpha)$ and $B(x)-B(\beta)$. Therefore, the common elements of $(A+\alpha)^{-1}$ and $B+\beta$ are roots of the polynomial

$$
x C(x)+A(\alpha) x^{q^{d-1}}\left(B(x)-x^{q^{d}}\right)+B(\beta) x^{q^{d-1}}\left(A(1 / x)-1 / x^{q^{d}}\right),
$$

and hence $\left|(A+\alpha)^{-1} \cap(B+\beta)\right| \leq 2|B| / q$. However, we will not consider affine subspaces of $\overline{\mathbb{F}_{q}}$ any further in this paper.

We conclude this section by mentioning an alternate, more direct proof of Csajbók's bound in the two-dimensional case. Let $A$ and $B$ be arbitrary twodimensional subspaces of $\overline{\mathbb{F}_{q}}$, and consider a maximal set of $\mathbb{F}_{q}$-linearly independent elements in $A^{-1} \cap B$. If that set is empty or a singleton, then $\left|A^{-1} \cap B\right|$ equals 0 or $q-1$. Otherwise, that set consists of $1 / \xi$ and $1 / \eta$, for some $\xi, \eta \in A$. If $\left|A^{-1} \cap B\right|>2 q-2$, then the inverse of some nontrivial linear combination of $\xi$ and $\eta$ also belongs to $B$. After possibly scaling $\xi$ or $\eta$ we may assume 
that $1 /(\xi+\eta)$ belongs to $B$, and hence $1 /(\xi+\eta)=a / \xi+b / \eta$ for some nonzero $a, b \in \mathbb{F}_{q}$. Therefore, $\xi / \eta$ satisfies a quadratic equation with coefficients in $\mathbb{F}_{q}$, and hence $\xi / \eta \in \mathbb{F}_{q^{2}}$. (A generalisation of this argument is presented in [Mat].) Consequently, $A$ is an $\mathbb{F}_{q^{2}}$-subspace, and $B=A^{-1}$ follows. We have shown that $\left|A^{-1} \cap B\right| /(q-1) \in\{0,1,2, q+1\}$.

In particular, this argument proves an easy fact which is mentioned right after [Csa13, Proposition 4.5]: any pair $(A, B)$ of two-dimensional $\mathbb{F}_{q^{-}}$subspaces of $\overline{\mathbb{F}_{q}}$ which attain equality in Csajbók's bound $\left|A^{-1} \cap B\right| \leq 2 q-2$, is obtained by taking as $A$ an arbitrary two-dimensional $\mathbb{F}_{q^{-}}$-subspace which is not an $\mathbb{F}_{q^{2}}$ subspace, and as $B$ the $\mathbb{F}_{q}$-span of the inverses of any two $\mathbb{F}_{q}$-linearly independent elements of $A$.

\section{A special COnfiguration of subspaces}

In this section we consider two $\mathbb{F}_{q}$-subspaces $A$ and $B$ of $\overline{\mathbb{F}_{q}}$, of dimension $d$, in a rather special configuration. That assumption insures that the polynomial $C(x)$ of Equation (2.1) has a factor of the form $x^{q^{d-1}-1}+c$, and this allows precise control over the set of roots of $C(x)$. This may seem like a rather artificial situation but, as we will see later in Theorem 9 , when $d=3$ it includes all cases where equality is attained in Csajbók's bound. Our proof is purely algebraic, but after the proof we will explain what goes on in geometric terms.

Because $\left(\gamma^{-1} A\right)^{-1} \cap \gamma B=\gamma\left(A^{-1} \cap B\right)$ for any $\gamma \in \overline{\mathbb{F}}_{q}^{*}$, we declare the ordered pair of $\mathbb{F}_{q^{-}}$-subspaces $\left(\gamma^{-1} A, \gamma B\right)$ to be equivalent to the pair $(A, B)$. Note that in principle one may consider a weaker equivalence relation which includes the application of field automorphisms, and possibly interchanging $A$ and $B$, but we chose not to do so.

Theorem 4. Let $A$ and $B$ be $\mathbb{F}_{q}$-subspaces of $\overline{\mathbb{F}_{q}}$ of size $q^{3}$, and suppose that $\left(A^{-1} \cap B\right) \cup\{0\}$ contains a one-dimensional $\mathbb{F}_{q^{2}}$-subspace of $\overline{\mathbb{F}_{q}}$. Then the pair $(A, B)$ is equivalent to a pair of subspaces consisting of the roots of the polynomials $A(x)=x^{q^{3}}+a x^{q^{2}}-x^{q}-a x$ and $B(x)=x^{q^{3}}+b x^{q^{2}}-x^{q}-b x$, for some $a, b \in{\overline{\mathbb{F}_{q}}}^{*}$, and we have

(1) $\left|A^{-1} \cap B\right|=2 q^{2}-2$ if $q$ is odd, $a^{q+1}=b^{q+1}=-1, a^{(q+1) / 2} \neq b^{(q+1) / 2}$, and $a b \neq 1$

(2) $\left|A^{-1} \cap B\right|=2 q^{2}-2 q$ if $q$ is odd, $a^{q+1}=-1$, and $a^{(q+1) / 2}=b^{(q+1) / 2}$;

(3) $\left|A^{-1} \cap B\right|=2 q^{2}-q-1$ if $q$ is even, $a^{q+1}=b^{q+1}=1$, and $a b \neq 1$;

(4) $\left|A^{-1} \cap B\right| \leq q^{2}+2 q-3$ otherwise.

Proof. With notation as in the proof of Theorem 1, let $A(x)=x^{q^{3}}+a_{2} x^{q^{2}}+$ $a_{1} x^{q}+a_{0} x$ and $B(x)=x^{q^{3}}+b_{2} x^{q^{2}}+b_{1} x^{q}+b_{0} x$ be the monic polynomials with distinct roots, hence with $a_{0} b_{0} \neq 0$, which have $A$ and $B$ as their sets of roots. 
That proof shows that all elements of $A^{-1} \cap B$ are roots of the polynomial

$$
\begin{aligned}
C(x)=- & a_{0} b_{2} x^{2 q^{2}-2}-a_{1} b_{2} x^{2 q^{2}-q-1}-a_{0} b_{1} x^{q^{2}+q-2} \\
& +\left(1-a_{2} b_{2}-a_{1} b_{1}-a_{0} b_{0}\right) x^{q^{2}-1} \\
& -a_{1} b_{0} x^{q^{2}-q}-a_{2} b_{1} x^{q-1}-a_{2} b_{0} .
\end{aligned}
$$

By hypothesis $\left(A^{-1} \cap B\right) \cup\{0\}$ contains a one-dimensional $\mathbb{F}_{q^{2}}$-subspace, hence the set of roots of a polynomial of the form $x^{q^{2}}+c x$. After replacing $A, B$ with an equivalent pair we may assume that $c=-1$, which means assuming that the

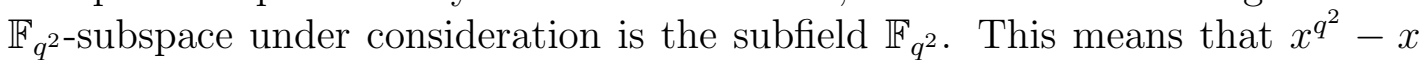
divides both $A(x)$ and $B(x)$, whence easily $A(x)=x^{q^{3}}+a x^{q^{2}}-x^{q}-a x$ and $B(x)=x^{q^{3}}+b x^{q^{2}}-x^{q}-b x$, and so

$$
\begin{aligned}
C(x)= & a b x^{2 q^{2}-2}+b x^{2 q^{2}-q-1}-a x^{q^{2}+q-2} \\
& -2 a b x^{q^{2}-1} \\
& \quad-b x^{q^{2}-q}+a x^{q-1}+a b . \\
= & \left(x^{q^{2}-1}-1\right)\left(a b x^{q^{2}-1}+b x^{q^{2}-q}-a x^{q-1}-a b\right) .
\end{aligned}
$$

Because all polynomials involved can be expressed as polynomials in $x^{q-1}$ we conveniently set $y=x^{q-1}$, and so

$$
C(x)=\left(y^{q+1}-1\right)\left(a b y^{q+1}+b y^{q}-a y-a b\right) .
$$

The derivative criterion shows that the second factor of $C(x)$ shown above has distinct roots unless $a b=1$, in which case it equals $\left(y^{q}-a\right)\left(y+a^{-1}\right)$, that is to say, $\left(y-a^{1 / q}\right)^{q}\left(y+a^{-1}\right)$. In that case $C(x)$ has at most $q^{2}+2 q-3$ distinct roots in $\overline{\mathbb{F}_{q}}$ (as a polynomial in $x$ ), and hence $\left|A^{-1} \cap B\right| \leq q^{2}+2 q-3$, as claimed in assertion (4) of the theorem. (This can be improved to $\left|A^{-1} \cap B\right| \leq q^{2}+q-2$ when $a^{q+1}= \pm 1$, because then the binomial $y+a^{-1}$ divides one of the other factors $y^{q+1}-1$ and $y^{q}-a$ of $C(x)$.)

Assume $a b \neq 1$ from now on. Because

$$
\left(y^{q+1}+a^{-1} y^{q}-b^{-1} y-1\right) \cdot y-\left(y^{q+1}-1\right) \cdot\left(y+a^{-1}\right)=-b^{-1} y^{2}+a^{-1}
$$

we see that the two exhibited factors of $C(x)$ are coprime unless $(b / a)^{(q+1) / 2}=1$ for $q$ odd, and unless $(b / a)^{q+1}=1$ for $q$ even, and their greatest common divisor equals $x^{2 q-2}-b / a$ in those cases. Note that this has distinct roots when $q$ is odd, but it has $q-1$ double roots when $q$ is even. This will account for the distinction between assertions (1), (2), and (3) of the theorem.

Our next task is to find the degree of the greatest common divisor of $x^{q^{3}} A(1 / x)$ and $C(x)$. To this goal we compute the remainder of the polynomial $x^{q^{3}} A(1 / x)$ modulo $C(x) /\left(x^{q^{2}-1}-1\right)$. All congruences in the remainder of the proof will tacitly be modulo the latter polynomial, that is, modulo its scalar multiple $y^{q+1}+$ 
$a^{-1} y^{q}-b^{-1} y-1$. We have

$$
\begin{aligned}
-b x^{q^{3}} A(1 / x) & =a b y^{q^{2}+q+1}+b y^{q^{2}+q}-a b y^{q^{2}}-b \\
& =\left(a b y^{q+1}+b y^{q}-a b\right) y^{q^{2}}-b \\
& \equiv a y \cdot y^{q^{2}}-b .
\end{aligned}
$$

Now note that $y^{q} \equiv\left(b^{-1} y+1\right) /\left(y+a^{-1}\right)$, where our assumption $a b \neq 0$ ensures that the denominator is coprime with the modulus. Consequently, we have

$$
y^{q^{2}} \equiv \frac{b^{-q} y^{q}+1}{y^{q}+a^{-q}} \equiv \frac{b^{-q} \frac{b^{-1} y+1}{y+a^{-1}}+1}{\frac{b^{-1} y+1}{y+a^{-1}}+a^{-q}}=\frac{\left(1+b^{-q-1}\right) y+\left(a^{-1}+b^{-q}\right)}{\left(a^{-q}+b^{-1}\right) y+\left(a^{-q-1}+1\right)} .
$$

Substituting this into our previous congruence we find

$$
\begin{aligned}
-b x^{q^{3}} A(1 / x) & \equiv a y \cdot \frac{\left(1+b^{-q-1}\right) y+\left(a^{-1}+b^{-q}\right)}{\left(a^{-q}+b^{-1}\right) y+\left(a^{-q-1}+1\right)}-b \\
& =\frac{a\left(1+b^{-q-1}\right) y^{2}+\left(a b^{-q}-a^{-q} b\right) y-\left(a^{-q-1}+1\right) b}{\left(a^{-q}+b^{-1}\right) y+\left(a^{-q-1}+1\right)} .
\end{aligned}
$$

Hence the greatest common divisor of $x^{q^{3}-1} A(1 / x)$ and $y^{q+1}+a^{-1} y^{q}-b^{-1} y-1$ divides the numerator of this expression.

If that numerator vanishes, that is, if $a^{q+1}=b^{q+1}=-1$, then the factor $y^{q+1}+a^{-1} y^{q}-b^{-1} y-1$ of $C(x)$ divides $x^{q^{3}} A(1 / x)$, and by assumption so does the other factor $y^{q+1}-1$ of $C(x)$. We conclude that the greatest common divisor of $x^{q^{3}} A(1 / x)$ and $B(x)$, which divides $C(x)$ but has distinct roots, equals the least common multiple of the two factors $x^{q^{2}-1}-1$ and $x^{q^{2}-1}+a^{-1} x^{q^{2}-q}-b^{-1} x^{q-1}-1$ of $C(x)$. According to an earlier calculation, when $q$ is odd this has degree $2 q^{2}-2$ if $a^{(q+1) / 2} \neq b^{(q+1) / 2}$, and $2 q^{2}-2 q$ otherwise, proving assertions (1) and (2) of the theorem. When $q$ is even it has degree $2 q^{2}-q-1$, as stated in assertion (3).

If the numerator of the expression found in Equation (3.1) does not vanish, then the greatest common divisor of $x^{q^{3}} A(1 / x)$ and $B(x)$ divides the product of that numerator and $x^{q^{2}-1}-1$, whence $\left|A^{-1} \cap B\right| \leq q^{2}+2 q-3$, as claimed in assertion (4).

We briefly pause to comment on the geometric interpretation of the intersection $A^{-1} \cap B$ in the case considered above, as a subset of the three-dimensional

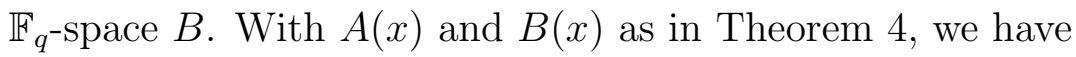

$$
x^{q+2} \cdot C(x)=\left(x^{q^{2}}-x\right)\left(a b x^{q^{2}+q}+b x^{q^{2}+1}-a x^{2 q}-a b x^{q+1}\right) .
$$

The monomials $x, x^{q}$ and $x^{q^{2}}$ determine $\mathbb{F}_{q^{-}}$linear maps $B \rightarrow \overline{\mathbb{F}_{q}}$, and so they uniquely extend to independent $\overline{\mathbb{F}_{q}}$-linear coordinates $x_{0}, x_{1}$ and $x_{2}$ on the linear space $B \otimes_{\mathbb{F}_{q}} \overline{\mathbb{F}_{q}}$. With this interpretation, the two factors in the above factorisation of $x^{q+2} \cdot C(x)$ may be viewed as representing a linear form and a quadratic form on $B \otimes_{\mathbb{F}_{q}} \overline{\mathbb{F}_{q}}$, namely, $x_{2}-x_{0}$ and $a b x_{1} x_{2}+b x_{0} x_{2}-a x_{1}^{2}-a b x_{0} x_{1}$. The 
quadratic form is nonsingular provided $a b \neq 1$, as we assume in the rest of this discussion.

In the projective plane $\mathbb{P}^{2}\left(\overline{\mathbb{F}_{q}}\right)$ associated with the linear space $B \otimes_{\mathbb{F}_{q}} \overline{\mathbb{F}_{q}}$, this means that the roots of $C(x)$ represent the $\mathbb{F}_{q}$-rational points of the union of a line, which is defined over $\mathbb{F}_{q}$, and a nonsingular conic, which may or may not be defined over $\mathbb{F}_{q}$.

The first three assertions of Theorem 4 correspond to the case where the conic is defined over $\mathbb{F}_{q}$, and the further distinction depends on whether the line is external, secant, or tangent to the conic, with the first two cases occurring only for $q$ odd, and the last case only for $q$ even. An alternate presentation of this configuration for $A^{-1} \cap B$, using ideas from finite geometries and limited to $q$ odd, is given in Section 4 of Csajbók's paper [Csa13].

To complete our geometric interpretation of Theorem 4, when the conic under consideration is not defined over $\mathbb{F}_{q}$, its $\mathbb{F}_{q}$-rational points belong also to the conic obtained from it by applying the Frobenius map $\alpha \mapsto \alpha^{q}$ to its coefficients, and so they are at most four, as they lie on the intersection of two distinct nonsingular conics. However, a simple calculation, of which we will sketch a more complex version for cubics in the proof of Theorem 7 , shows that at most two of the intersection points of the conics over $\overline{\mathbb{F}_{q}}$ are $\mathbb{F}_{q}$-rational. Adding to those the number of points on the line provides a geometric interpretation for the bound $\left|A^{-1} \cap B\right| /(q-1) \leq q+3$ obtained for that case in Theorem 4 .

Part of the argument in the proof of Theorem 4 applies to pairs of higherdimensional subspaces in a special configuration described in the following result, which will be needed later, in the proof of Theorem 2 .

Theorem 5. Let $A$ and $B$ be $\mathbb{F}_{q}$-subspaces of $\overline{\mathbb{F}_{q}}$ of size $q^{d} \geq q^{3}$, and suppose

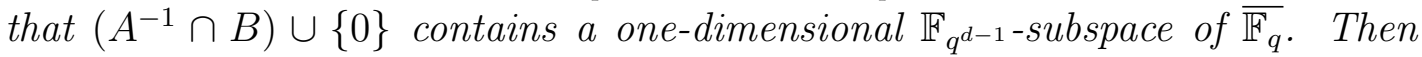
$\left|A^{-1} \cap B\right| \leq q^{d-1}+2 q^{2}-3$.

Proof. We argue in a similar way as in the proof of Theorem 4. After replacing $A, B$ with an equivalent pair we may assume that $x^{q^{d}}-x$ divides both $A(x)$ and $B(x)$, whence $A(x)=x^{q^{d}}+a x^{q^{d-1}}-x^{q}-a x$ and $B(x)=x^{q^{d}}+b x^{q^{d-1}}-x^{q}-b x$, with $a b \neq 0$, and so

$$
C(x)=\left(x^{q^{d-1}-1}-1\right)\left(a b x^{q^{d-1}-1}+b x^{q^{d-1}-q}-a x^{q-1}-a b\right) .
$$

We shall compute the remainder of the polynomial $x^{q^{4}} A(1 / x)$ modulo the second factor of $C(x)$. Working modulo that polynomial we have

$$
\begin{aligned}
-b x^{q^{d}} A(1 / x) & =a b x^{q^{d}-1}+b x^{q^{d}-q}-a b x^{q^{d}-q^{d-1}}-b \\
& =\left(a b x^{q^{d-1}-1}+b x^{q^{d-1}-q}-a b\right) x^{q^{d}-q^{d-1}}-b \\
& \equiv a x^{q-1} \cdot x^{q^{d}-q^{d-1}}-b .
\end{aligned}
$$


Now we conveniently set $y=x^{q-1}$. Because $y^{q^{d-2}+\cdots+q} \equiv\left(b^{-1} y+1\right) /\left(y+a^{-1}\right)$, we have

$$
y^{q^{d-1}+\cdots+q^{2}} \equiv \frac{b^{-q} y^{q}+1}{y^{q}+a^{-q}},
$$

and hence

$$
\begin{aligned}
-b x^{q^{d}} A(1 / x) & \equiv a y \cdot y^{q^{d-1}}-b \\
& =\frac{1}{y^{q^{d-2}+\cdots+q}}\left(a y \cdot y^{q} \cdot y^{q^{d-1}+\cdots+q^{2}}\right)-b \\
& \equiv a y^{q+1} \cdot \frac{y+a^{-1}}{b^{-1} y+1} \cdot \frac{b^{-q} y^{q}+1}{y^{q}+a^{-q}}-b \\
& \equiv \frac{a b^{-q} y^{2 q+2}+b^{-q} y^{2 q+1}+a y^{q+2}-b y^{q}-a^{-q} y-b a^{-q}}{\left(b^{-1} y+1\right)\left(y^{q}+a^{-q}\right)} .
\end{aligned}
$$

Because the numerator of this expression is nonzero, its degree $(2 q+2)(q-1)=$ $2 q^{2}-2$, in the original indeterminate $x$, is an upper bound for the degree of the greatest common divisor of $x^{q^{d-1}-1}+a^{-1} x^{q^{d-1}-q}-b^{-1} x^{q-1}-1$ and $x^{q^{d}} A(1 / x)$. The desired conclusion follows by taking the other factor $x^{q^{d-1}-1}-1$ of $C(x)$ into account.

\section{A BetTER BOUND FOR SUBSPACES OF DIMENSION AT LEAST FOUR}

The proof of Theorem 1 which we gave in Section 2 shows that all elements of $A^{-1} \cap B$ are roots of the polynomial $C(x)$ of Equation (2.1), and hence of the modified polynomial

$$
\begin{aligned}
C_{0}(x) & =x^{1+\left(1+q+q^{2}+\cdots+q^{d-2}\right)} \cdot C(x) \\
& =x^{1+q+q^{2}+\cdots+q^{d-1}}\left(1-\left(\sum_{i=0}^{d-1} a_{i} / x^{q^{i}}\right) \cdot\left(\sum_{j=0}^{d-1} b_{j} x^{q^{j}}\right)\right) .
\end{aligned}
$$

The latter has the advantage of being a linear combination of monomials, each of whose degrees is a sum of $d$ terms taken from the set $\left\{1, q, \ldots, q^{d-1}\right\}$ with at most one repetition, and hence one omission. We now show how being a root of $C_{0}(x)$ can be interpreted as being a zero of one or more homogeneous forms of degree $d$ on the $\mathbb{F}_{q^{-} \text {-space } B \text {. }}$

The $\mathbb{F}_{q^{-}}$-linear maps $x_{i}: B \rightarrow \overline{\mathbb{F}_{q}}$ given by $x \mapsto x^{q^{i}}$, for $0 \leq i<d$, are $\mathbb{F}_{q^{-}}$ linearly independent, and so they form a complete set of linear coordinates (that is, a basis of the dual space) on the $\overline{\mathbb{F}_{q}}$-space $B \otimes_{\mathbb{F}_{q}} \overline{\mathbb{F}_{q}}$. Hence to $C_{0}(x)$ there corresponds a homogeneous polynomial function

$$
E\left(x_{0}, \ldots, x_{d-1}\right)=x_{0} \cdots x_{d-1} \cdot\left(1-\left(\sum_{i=0}^{d-1} a_{i} / x_{i}\right) \cdot\left(\sum_{j=0}^{d-1} b_{j} x_{j}\right)\right),
$$

of degree $d$, defined on the $\overline{\mathbb{F}_{q}}$-space $B \otimes_{\mathbb{F}_{q}} \overline{\mathbb{F}_{q}}$. We would rather need a polynomial function on the original $\mathbb{F}_{q}$-space $B$, but we can obtain that through a linear 
change of coordinates. In fact, an arbitrary $\mathbb{F}_{q}$-linear map on $B$ with values in $\mathbb{F}_{q}$ is given by

$$
\begin{aligned}
x \mapsto b_{0} \gamma x+\left(b_{0}^{q} \gamma^{q}+b_{1} \gamma\right) x^{q}+\left(b_{0}^{q^{2}} \gamma^{q^{2}}+b_{1}^{q} \gamma^{q}+b_{2} \gamma\right) x^{q^{2}}+\cdots \\
\cdots+\left(b_{0}^{q^{d-1}} \gamma^{q^{d-1}}+b_{1}^{q^{d-2}} \gamma^{q^{d-2}}+\cdots+b_{d-1} \gamma\right) x^{q^{d-1}}
\end{aligned}
$$

where $\gamma$ ranges over the roots of the $q$-polynomial $b_{0}^{q^{d}} x^{q^{d}}+b_{1}^{q^{d-1}} x^{q^{d-1}}+\cdots+b_{d} x$. Therefore, a complete set of $\mathbb{F}_{q}$-linear coordinates $z_{0}, \ldots, z_{d-1}$ on the $\mathbb{F}_{q}$-space $B$ is obtained by letting $\gamma$ range over an $\mathbb{F}_{q}$-basis of the roots of that $q$-polynomial. After expressing each $x_{i}$ in terms of $z_{0}, \ldots, z_{d-1}$ (as linear combinations over $\left.\overline{\mathbb{F}_{q}}\right)$, the polynomial function $E\left(x_{0}, \ldots, x_{d-1}\right)$ on $B \otimes_{\mathbb{F}_{q}} \overline{\mathbb{F}_{q}}$ yields a polynomial function $\tilde{E}\left(z_{0}, \ldots, z_{d-1}\right)$ on $B$, but still with values in $\frac{\mathbb{F}_{q}}{}$, which is homogeneous of degree $d$ in $z_{0}, \ldots, z_{d-1}$.

To recapitulate in slightly different wording, all elements of $A^{-1} \cap B$, once $B$ is identified with $\mathbb{F}_{q}^{d}$ via the coordinates $z_{i}$, are roots of the polynomial $\tilde{E}\left(z_{0}, \ldots, z_{d-1}\right) \in \overline{\mathbb{F}}_{q}\left[z_{0}, \ldots, z_{d-1}\right]$. According to Theorem 6 below, this polynomial turns out to be usually irreducible for our purposes, with notable exceptions where our geometric problem has already been dealt with in Section 3. When the polynomial is indeed irreducible, it defines a hypersurface in the projective space $\mathbb{P}\left(B \otimes_{\mathbb{F}_{q}} \overline{\mathbb{F}_{q}}\right) \cong \mathbb{P}^{d-1}\left(\overline{\mathbb{F}_{q}}\right)$, whose number of $\mathbb{F}_{q}$-rational points can be bounded using the Lang-Weil bound [LW54]. These are the ideas at play in the proof of our Theorem 2 stated in the Introduction, which we give below, but not before stating the result on the possible factorisations of $E$ which we have just mentioned.

Theorem 6. The homogeneous polynomial

$$
E=E\left(x_{1}, \ldots, x_{n}\right)=x_{1} \cdots x_{n} \cdot\left(1+\left(\sum_{i=1}^{n} a_{i} / x_{i}\right) \cdot\left(\sum_{j=1}^{n} b_{j} x_{j}\right)\right)
$$

where $a_{i}, b_{j} \in \overline{\mathbb{F}_{q}}$, has at most two non-monomial (absolutely) irreducible factors. If it has two then at least one of them is a linear combination of exactly two of the indeterminates.

Furthermore, if $E$ has $x_{1}+x_{2}$ as a factor, then either

$$
\begin{array}{r}
E /\left(x_{3} \cdots x_{n}\right)=x_{1} x_{2} \cdot\left(1+\left(a / x_{1}+(a+c) / x_{2}\right) \cdot\left(b x_{1}+\left(b-c^{-1}\right) x_{2}\right)\right) \\
=\left(x_{1}+x_{2}\right)\left((a+c) b x_{1}+a\left(b-c^{-1}\right) x_{2}\right)
\end{array}
$$

for some $a, b, c \in \overline{\mathbb{F}_{q}}$ with $c \neq 0$, or

$$
\begin{array}{r}
E /\left(x_{4} \cdots x_{n}\right)=x_{1} x_{2} x_{3} \cdot\left(1+\left(a / x_{1}+a / x_{2}-1 / x_{3}\right) \cdot\left(b x_{1}+b x_{2}-x_{3}\right)\right) \\
=\left(x_{1}+x_{2}\right)\left(a b x_{2} x_{3}+a b x_{1} x_{3}+b x_{1} x_{2}-a x_{3}^{2}\right),
\end{array}
$$

up to permuting the indeterminates $x_{3}, \ldots, x_{n}$.

The harmless sign change in the definition of $E$ from the previous notation will avoid the occurrence of several minus signs in the proof of Theorem 6 . We 
have also conveniently shifted the indices of the indeterminates, which now start from one. The proof of Theorem 6 is a little technical, and we postpone it to Section 6 to avoid disrupting the flow of the present argument. Note that the second nontrivial factorisation allowed by Theorem 6 has already occurred in disguise in the factorisations of $C(x)$ obtained in the proofs of Theorems 4 and 5 .

Proof of Theorem 2. Continue with the setting introduced above. As we noted in the proof of Theorem 1, the condition $A^{-1} \nsubseteq B$ implies that $C(x)$ is not the zero polynomial, whence $E\left(x_{0}, \ldots, x_{d-1}\right)$ is not the zero polynomial. Monomial factors of $E\left(x_{0}, \ldots, x_{d-1}\right)$ and, correspondingly, of $C(x)$, clearly give no contribution to estimating $\left|A^{-1} \cap B\right|$.

Suppose first that $E\left(x_{0}, \ldots, x_{d-1}\right)$ has a unique non-monomial irreducible factor $F\left(x_{0}, \ldots, x_{d-1}\right)$, of degree $d^{\prime} \leq d$. Let $\tilde{F}\left(z_{0}, \ldots, z_{d-1}\right)$ be the corresponding polynomial written in terms of the coordinates $z_{0}, \ldots, z_{d-1}$ on the $\mathbb{F}_{q}$-space $B$. It defines an irreducible algebraic subvariety of the projective space $\mathbb{P}\left(B \otimes_{\mathbb{F}_{q}} \overline{\mathbb{F}_{q}}\right) \cong \mathbb{P}^{d-1}\left(\overline{\mathbb{F}_{q}}\right)$, of dimension $d-2$ (that is, a hypersurface).

If that variety is defined over $\mathbb{F}_{q}$, which occurs if $\tilde{F}\left(z_{0}, \ldots, z_{d-1}\right)$, after multiplication by a suitable scalar, can be made to have all coefficients in $\mathbb{F}_{q}$, then according to the Lang-Weil estimate [LW54] its number of $\mathbb{F}_{q}$-rational points is bounded above by

$$
q^{d-2}+\left(d^{\prime}-1\right)\left(d^{\prime}-2\right) q^{d-(5 / 2)}+C_{d, d^{\prime}} \cdot q^{d-3},
$$

where $C_{d, d^{\prime}}$ is a constant which depends only on $d$ and $d^{\prime}$. The desired conclusion is then obtained upon multiplication by $q-1$ and using the fact that $d^{\prime} \leq d$.

Now suppose that the variety under consideration is not defined over $\mathbb{F}_{q}$. Then the Galois-conjugate polynomial $\tilde{F}^{\sigma}\left(z_{0}, \ldots, z_{d-1}\right)$, obtained from $\tilde{F}\left(z_{0}, \ldots, z_{d-1}\right)$ by applying the Frobenius automorphism $\sigma: a \mapsto a^{q}$ to each coefficient, is not proportional to $\tilde{F}\left(z_{0}, \ldots, z_{d-1}\right)$, and hence together with the latter it defines a (possibly reducible) algebraic set in $\mathbb{P}^{d-1}\left(\overline{\mathbb{F}_{q}}\right)$, of dimension strictly smaller than $d-2$, and degree at most $\left(d^{\prime}\right)^{2}$. According to a standard fact known as the Schwartz-Zippel lemma, see [EOT10, Lemma A.3] for a proof, the number of $\mathbb{F}_{q^{-}}$ rational points of this algebraic set is at most $\left(d^{\prime}\right)^{2}(q+1)^{d-3}$. After multiplying by $q-1$ we see that the desired conclusion holds in this case as well.

Now we may assume that $E\left(x_{0}, \ldots, x_{d-1}\right)$ has at least two non-monomial irreducible factors. Then it has exactly two according to Theorem 6 , and one of them is a linear combination of two of the indeterminates, say $x_{i}$ and $x_{j}$ with $i<j$. The corresponding factor of our original polynomial $C(x)$ is then a linear combination of $x^{q^{i}-1}$ and $x^{q^{j}-1}$, and hence it accounts for at most $q^{j-i}-1$ distinct nonzero roots of that polynomial. The possible factorisations of $E$ given in Theorem 6 show that the remaining factor of $C(x)$ has degree at most $q^{d-1}-1$, whence $\left|A^{-1} \cap B\right| \leq q^{d-1}+q^{j-i}-2$. If $j-i<d-1$ we have reached our goal, hence assume $j-i=d-1$. This means that the former factor of $C(x)$ considered above is a linear combination of 1 and $x^{q^{d-1}-1}$. Possibly after replacing $(A, B)$ with an equivalent pair we may assume that linear combination to be $x^{q^{d-1}-1}-1$. 
Now consider, in turn, the two possible factorisations of $E$ stated in Theorem 6, and what they entail for the polynomials $A(x)$ and $B(x)$ in our setting. The former factorisation implies $A(x)=x^{q^{d}}+a x^{q^{d-1}}-(a+c) x$ and $B(x)=x^{q^{d}}-$ $b x^{q^{d-1}}+\left(b-c^{-1}\right) x$, whence

$$
C(x)=\left(x^{q^{d-1}-1}-1\right)\left(-(a+c) b x^{q^{d-1}-1}+a\left(b-c^{-1}\right)\right) .
$$

Because $B(x) \equiv x^{q}-c^{-1} x\left(\bmod x^{q^{d-1}-1}-1\right)$, the polynomial $B(x)$ has at most $q-1$ nonzero roots in common with the former factor of $C(x)$, and similarly with the latter factor. Hence in this case we have $\left|A^{-1} \cap B\right| \leq 2 q-2$.

The other possible factorisation of $E$ yields $A(x)=x^{q^{d}}+a x^{q^{d-1}}-c x^{q^{e}}-a x$ and $B(x)=x^{q^{d}}+b x^{q^{d-1}}-c^{-1} x^{q^{e}}-b x$, with $0<e<d-1$ and $a b c \neq 0$, and so

$$
C(x)=\left(x^{q^{d-1}-1}-1\right)\left(a b x^{q^{d-1}-1}+b c x^{q^{d-1}-q^{e}}-a c^{-1} x^{q^{e}-1}-a b\right) .
$$

Because $B(x) \equiv x^{q}-c^{-1} x^{q^{e}}\left(\bmod x^{q^{d-1}-1}-1\right)$, the polynomial $B(x)$ has at most $q-1$ nonzero roots in common with the former factor of $C(x)$, whence $\left|A^{-1} \cap B\right| \leq q^{d-1}+q-2$, except when $c=1$ and $e=1$. However, in the latter case Theorem 5 applies and yields $\left|A^{-1} \cap B\right| \leq q^{d-1}+2 q^{2}-3$.

\section{A CLASSIFICATION OF PAIRS OF THREE-DIMENSIONAL SUBSPACES WITH LARGE INTERSECTION $A^{-1} \cap B$}

In the case of subspaces of dimension $d=3$, which was excluded from Theorem 2, parts of its proof still apply. In particular, the Lang-Weil estimate of Equation (4.1) takes the more precise form of the Hasse-Weil bound, and allows us to prove the following result.

Theorem 7. Let $A$ and $B$ be $\mathbb{F}_{q}$-subspaces of $\overline{\mathbb{F}_{q}}$, of size $q^{3}$, with $A^{-1} \nsubseteq B$. If $\left|A^{-1} \cap B\right| /(q-1)>q+1+\lfloor 2 \sqrt{q}\rfloor$, then $\left|A^{-1} \cap B\right| /(q-1)$ equals either $2 q+2$ or $2 q$ for $q$ odd, and it equals $2 q+1$ for $q$ even.

Note that equality in Csajbók's bound $\left|A^{-1} \cap B\right| \leq 2 q^{2}-2$ for three-dimensional spaces cannot be attained in characteristic two.

Proof. Arguing as in the proof of Theorem 2, which we gave in Section 4, and aiming at a contradiction, suppose that $E\left(x_{0}, x_{1}, x_{2}\right)$ is irreducible. Writing this in terms of the coordinates $z_{0}, \ldots, z_{d-1}$ on the $\mathbb{F}_{q}$-space $B$ we get a polynomial $\tilde{E}\left(z_{0}, z_{1}, z_{2}\right)$ which defines an irreducible cubic in the projective plane $\mathbb{P}\left(B \otimes_{\mathbb{F}_{q}}\right.$ $\left.\overline{\mathbb{F}_{q}}\right) \cong \mathbb{P}^{2}\left(\overline{\mathbb{F}_{q}}\right)$. If the cubic is defined over $\mathbb{F}_{q}$, then according to the Hasse-Weil bound its number of $\mathbb{F}_{q}$-rational points does not exceed $q+1+2 \sqrt{q}$, whence $\left|A^{-1} \cap B\right| /(q-1) \leq q+1+2 \sqrt{q}$, which contradicts our hypothesis. If the cubic is not defined over $\mathbb{F}_{q}$, then its intersection with the irreducible cubic defined by $\tilde{E}^{\sigma}\left(z_{0}, z_{1}, z_{2}\right)$, where $\sigma$ is the Frobenius automorphism, has at most $3^{2}=9$ points in $\mathbb{P}\left(B \otimes_{\mathbb{F}_{q}} \overline{\mathbb{F}_{q}}\right)$ according to Bézout's theorem (or to the SchwartzZippel lemma if we prefer). Hence $\left|A^{-1} \cap B\right| /(q-1) \leq 9$, and we obtain a contradiction because this number does not exceed Weil's bound $q+1+\lfloor 2 \sqrt{q}\rfloor$, 
except when $q \leq 3$. However, when $q=2$ Theorem 1 provides the improved bound $\left|A^{-1} \cap B\right| \leq 3 \cdot 2^{3} / 4-1=5$, which yields the desired contradiction.

In order to cover the case $q=3$ as well, we sketch how an explicit calculation allows us to strengthen the upper bound of 9 given by Bézout's theorem to the bound $\left|A^{-1} \cap B\right| /(q-1) \leq 6$, for arbitrary $q$. We do that by showing that the intersection of the zero sets of $\tilde{E}\left(z_{0}, z_{1}, z_{2}\right)$ and its Galois-conjugate $\tilde{E}^{\sigma}\left(z_{0}, z_{1}, z_{2}\right)$ contains at least three non-rational points. One way of computing the Galoisconjugate in terms of the original coordinates $x_{0}, x_{1}, x_{2}$ is raising the polynomial

$$
\begin{aligned}
C_{0}(x)=x^{q+2} \cdot C(x)=- & a_{0} b_{2} x^{2 q^{2}+q}-a_{1} b_{2} x^{2 q^{2}+1}-a_{0} b_{1} x^{q^{2}+2 q} \\
& +\left(1-a_{2} b_{2}-a_{1} b_{1}-a_{0} b_{0}\right) x^{q^{2}+q+1} \\
& -a_{1} b_{0} x^{q^{2}+2}-a_{2} b_{1} x^{2 q+1}-a_{2} b_{0} x^{q+2}
\end{aligned}
$$

to the $q$-th power and reducing the result modulo the polynomial $B(x)$. Writing both the remainder of this division and the original polynomial $C_{0}(x)$ in terms of $x_{0}=x, x_{1}=x^{q}$, and $x_{2}=x^{q^{2}}$, one discovers that both vanish for $\left(x_{0}, x_{1}, x_{2}\right)=$ $(1,0,0),\left(b_{1},-b_{0}, 0\right),\left(b_{2}, 0,-b_{0}\right)$. However, a triple $\left(x_{0}, x_{1}, x_{2}\right)$ gives a rational point of our curve, that is, an element of $B$, only when $x_{1}=x_{0}^{q}, x_{2}=x_{1}^{q}$, and $x_{2}^{q}=-b_{2} x_{2}-b_{1} x_{1}-b_{0} x_{0}$, which is not the case for any of the three triples found.

Thus, we have shown that $E\left(x_{0}, x_{1}, x_{2}\right)$ cannot be irreducible. Invoking Theorem 6 and arguing as in the proof of Theorem 2 we see that $\left|A^{-1} \cap B\right|$ can possibly be so large only when $C(x)$ has a non-trivial linear combination of 1 and $x^{q^{2}}$ as a factor, which may be taken to be $x^{q^{2}-1}-1$ after passing to an equivalent pair $(A, B)$. The proof of Theorem 2 also shows that we must have the second exceptional factorisation of $E\left(x_{0}, x_{1}, x_{2}\right)$ given in Theorem 6 , and that $A(x)=x^{q^{3}}+a x^{q^{2}}-x^{q}-a x$ and $B(x)=x^{q^{3}}+b x^{q^{2}}-x^{q}-b x$. Consequently, both $A$ and $B$ contain $\mathbb{F}_{q^{2}}$, hence Theorem 4 applies and completes the proof.

Remark 8. A special version of Theorem 7 occurs as assertion (1) of [Csa13, Theorem 4.8]. That result restricts $A$ and $B$ to be contained in $\mathbb{F}_{q^{4}}$, which is a rather strong assumption. Note that [Csa13, Theorem 4.8] is actually proved under the unstated hypothesis that $q$ is odd, but fails to exclude the possibility that $\left|A^{-1} \cap B\right| /(q-1)=2 q+1$ (except in the special case where $A=B$ ). At the author's request Csajbók has produced a proof which excludes that possibility, based on similar methods as [Csa13].

Theorem 7, combined with the special configuration which we investigated in Theorem 4, allows us to classify all pairs $(A, B)$ attaining equality in Csajbók's bound, or almost, in the three-dimensional case.

Theorem 9. In the following assertions $A$ and $B$ denote $\mathbb{F}_{q}$-subspaces of $\overline{\mathbb{F}_{q}}$, of size $q^{3}$, with $A^{-1} \nsubseteq B$.

(1) There are exactly $(q-1) / 2$ equivalence classes of pairs $(A, B)$ such that $\left|A^{-1} \cap B\right|=2 q^{2}-2$.

(2) For odd $q>5$ there are exactly $(q+1) / 2$ equivalence classes of pairs $(A, B)$ such that $\left|A^{-1} \cap B\right|=2 q^{2}-2 q$. 
(3) For even $q>4$ there are exactly $q$ equivalence classes of pairs $(A, B)$ such that $\left|A^{-1} \cap B\right|=2 q^{2}-q-1$.

(4) Each equivalence class described in assertions (1) and (2) contains exactly two pairs satisfying $A=B$, and each equivalence class described in assertion (3) contains exactly one such pair. Each such subspace $A=B$ is contained in $\mathbb{F}_{q^{4}}$, and equals the kernel of $x \mapsto \operatorname{Tr}_{\mathbb{F}_{q^{4}} / \mathbb{F}_{q}}(\alpha x)$, for some $\alpha \in \mathbb{F}_{q^{4}}$ with $\alpha^{2} \in \mathbb{F}_{q^{2}} \backslash \mathbb{F}_{q}$.

Proof. The stated conditions on $q$ insure that $\left|A^{-1} \cap B\right| /(q-1)$ exceeds the Hasse-Weil bound in each case. Therefore, as in the proof of Theorem 7 we conclude that after replacing $(A, B)$ with an equivalent pair we have $A(x)=$ $x^{q^{3}}+a x^{q^{2}}-x^{q}-a x$ and $B(x)=x^{q^{3}}+b x^{q^{2}}-x^{q}-b x$, and Theorem 4 gives exact conditions that $a$ and $b$ satisfy.

Now the subspaces $\gamma^{-1} A$ and $\gamma B$, which form an equivalent pair to $(A, B)$ for $\gamma \in \overline{\mathbb{F}}_{q}^{*}$, are the sets of roots of the monic polynomials $A_{\gamma^{-1}}(x)=\gamma^{-q^{3}} A(\gamma x)$ and $B_{\gamma}(x)=\gamma^{q^{3}} B(x / \gamma)$. Comparing coefficients we see that each of the equalities $\gamma^{-1} A=A$ and $\gamma B=B$ occurs only when $\gamma^{q-1}=1$, that is, when $\gamma \in \mathbb{F}_{q}^{*}$. However, $A_{\gamma^{-1}}(x)$ has the admissible form $A_{\gamma^{-1}}(x)=x^{q^{3}}+a^{\prime} x^{q^{2}}-x^{q}-a^{\prime} x$ considered above (which means that it is a multiple of $x^{q^{2}}-1$ ) if and only if $\gamma \in \mathbb{F}_{q^{2}}^{*}$, and so does $B_{\gamma}(x)$. Consequently, each equivalence class of pairs $(A, B)$ under consideration contains exactly $q+1$ pairs for which the corresponding polynomials $A(x)$ and $B(x)$ have the required form. Thus, the number of equivalence classes is obtained after dividing by $q+1$ the number of pairs $(a, b)$ with $a^{q+1}=b^{q+1}=-1$ and $a b \neq 1$, and possibly the further conditions given in Theorem 4. This establishes assertions (1), (2), and (3).

A similar coefficient comparison shows that for the pairs $(A, B)$ under consideration an equivalent pair $\left(\gamma^{-1} A, \gamma B\right)$ satisfies $\gamma^{-1} A=\gamma B$ exactly when $\gamma^{2(q-1)}=a / b$. Consequently, there are two such pairs equivalent to $(A, B)$ when $q$ is odd, and only one when $q$ is even, as claimed in assertion (4). Furthermore, taking $\gamma^{2(q-1)}=a / b$, whence $\gamma^{q^{2}-1}=(a / b)^{(q+1) / 2}= \pm 1$, we obtain

$$
\begin{aligned}
A_{\gamma^{-1}}(x)=B_{\gamma}(x) & =x^{q^{3}}+\gamma^{q^{3}-q^{2}} b x^{q^{2}}-\gamma^{q^{3}-q} x^{q}+\gamma^{q^{3}-1} b x \\
& =x^{q^{3}}+\gamma^{q-1} b x^{q^{2}}-\gamma^{q^{2}-1}\left(x^{q}+\gamma^{q-1} b x\right) \\
& =x^{q^{3}}+c x^{q^{2}}+c^{q+1}\left(x^{q}+c x\right) \\
& =x^{q^{3}}+c x^{q^{2}}+c^{q+1} x^{q}+c^{q^{2}+q+1} x,
\end{aligned}
$$

having set $c=\gamma^{q-1} b$ and noted that $c^{q+1}=-\gamma^{q^{2}-1}= \pm 1$. For $(a, b)$ ranging over all pairs such that $a^{q+1}=b^{q+1}=-1$ and $a b \neq 1$, and with $\gamma$ chosen as above, $c$ takes all the values such that $c^{2(q+1)}=1$ and $c^{2} \neq 1$. The description of $A$ given in assertion (4) follows at once by writing $c=\alpha^{-(q-1)}$.

Remark 10. The restrictions on $q$ in assertions (2) and (3) of Theorem 9 cannot be relaxed. For example, a computer calculation shows that $\mathbb{F}_{5^{4}}$ contains $31 \cdot 8$ pairs $(A, B)$ of three-dimensional $\mathbb{F}_{5}$-subspaces such that $\left|A^{-1} \cap B\right|=2 q^{2}-2 q=40$, 
rather than $\left(q^{2}+q+1\right) \cdot(q+1) / 2=31 \cdot 3$ as Theorem 9 would predict. For those in excess, $A^{-1} \cap B$ yields an irreducible cubic in the projective plane $\mathbb{P} B$.

Remark 11. In case of assertion (1) of Theorem 9, where equality in Csajbók's bound is attained, an alternate approach is available and described in [Mat]. It relies on facts from finite geometries to bypass the arguments of this and the previous section, and hence Theorem 6 , on which they ultimately depend. Briefly, as a special case of a more general result it is shown in [Mat] that the image of $A^{-1} \cap B$ in $\mathbb{P} B$ is an arc, for three-dimensional $\mathbb{F}_{q^{-}}$subspaces $A, B$ of $\overline{\mathbb{F}_{q}}$,

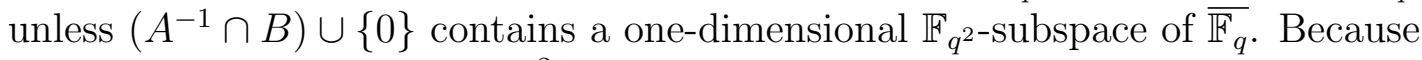
it is known that an arc in $\mathbb{P}^{2}\left(\mathbb{F}_{q}\right)$ has at most $2 q+1$ points if $q>3$, when equality $\left|A^{-1} \cap B\right| /(q-1)=2 q+2$ holds in Csajbók's bound we conclude that

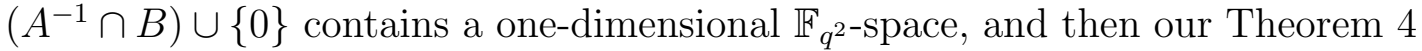
applies. If $q$ is odd (and larger than three), at this point one can also deduce that the image of $A^{-1} \cap B$ in $\mathbb{P} B$ is the union of a line and a conic without using Theorem 4, appealing instead to the classical result of B. Segre that an an arc with $q+1$ points in a projective plane is a conic (for $q$ odd), see [Mat, Theorem 5].

Remark 12. In contrast with the three-dimensional case considered in Theorem 9, for a fixed prime power $q$ there are infinitely many equivalence classes of pairs $(A, B)$ of two-dimensional $\mathbb{F}_{q^{-}}$subspaces which attain equality in Csajbók's bound $\left|A^{-1} \cap B\right| \leq 2 q-2$. This follows at once from their description which we gave at the end of Section 2.

Corollary 13. Assume $q>5$, and set $P(x)=x^{q^{3}}+x^{q^{2}}+x^{q}+x$. If $A$ and $B$ are $\mathbb{F}_{q}$-subspaces of $\overline{\mathbb{F}_{q}}$, of size $q^{3}$, such that

$$
q^{2}-1+\left\lfloor 2 q^{1 / 2}\right\rfloor \cdot(q-1)<\left|A^{-1} \cap B\right|<q^{3}-1,
$$

then $A$ and $B$ are the sets of roots of $P\left(\alpha \gamma^{-1} x\right)$ and $P(\alpha \gamma x)$, respectively, for some $\alpha, \gamma \in{\overline{\mathbb{F}_{q}}}^{*}$ with $\alpha^{2} \in \mathbb{F}_{q^{2}} \backslash \mathbb{F}_{q}$.

Proof. According to Theorem 7 the pair $(A, B)$ is one of those described in Theorem 9. According to assertion (4) of the latter, some equivalent pair $\left(\gamma^{-1} A, \gamma B\right)$ satisfies $\gamma^{-1} A=\gamma B$, and that subspace equals the set of roots of $P(\alpha x)$, for some $\alpha \in{\overline{\mathbb{F}_{q}}}^{*}$ with $\alpha^{2} \in \mathbb{F}_{q^{2}} \backslash \mathbb{F}_{q}$.

The explicit description of the spaces $A$ and $B$ given in Corollary 13 allows one to decide whether and how many of them can be found inside a given finite field. For example, in $\mathbb{F}_{q^{4}}$ there are exactly $2 q\left(q^{2}+q+1\right)$ such pairs $(A, B)$ for $q$ odd, and $q\left(q^{2}+q+1\right)$ for $q$ even, because $\gamma \in \mathbb{F}_{q^{4}}^{*}$ in this case. Those among them with $A=B$ are in number of $2 q$ and $q$, respectively, and for $q$ odd they match those described in [Csa13, Propositions 4.4 and 4.5].

Another example is the following improvement of Csajbók's bound for threedimensional subspaces of finite fields which do not contain $\mathbb{F}_{q^{4}}$.

Corollary 14. Consider the finite field $\mathbb{F}_{q^{e}}$, where $q>5$ and $e$ is not a multiple of four. If $A$ and $B$ are $\mathbb{F}_{q^{-}}$-subspaces of $\mathbb{F}_{q^{e}}$ with size $q^{3}$, and $A^{-1} \nsubseteq B$, then $\left|A^{-1} \cap B\right| \leq q^{2}-1+\left\lfloor 2 q^{1 / 2}\right\rfloor \cdot(q-1)$. 
Proof. Consider subspaces $A$ and $B$ of $\overline{\mathbb{F}_{q}}$ as in Corollary 13. When $\gamma=1$ they are both contained in $\mathbb{F}_{q^{4}}$, and $\left(A^{-1} \cap B\right) \cup\{0\}$ properly contains the one-

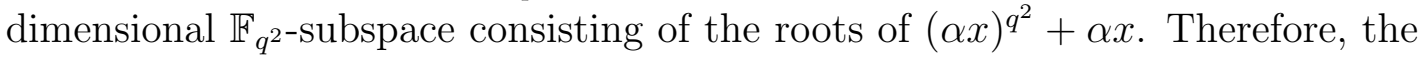
subfield generated by all the quotients of pairs of elements of $A^{-1} \cap B$ properly contains $\mathbb{F}_{q^{2}}$, and hence equals $\mathbb{F}_{q^{4}}$. This last statement carries over to the case of arbitrary $\gamma$. Consequently, the subfield of $\overline{\mathbb{F}_{q}}$ generated by $A^{-1} \cap B$ contains $\mathbb{F}_{q^{4}}$, and hence $A$ and $B$ cannot be both contained in $\mathbb{F}_{q^{e}}$.

\section{Proof of Theorem 6}

Recall that a polynomial is called multilinear if it has degree at most one in each indeterminate. The following property of the polynomial $E$, which follows from the definition and is inherited by factors, will be crucial in most of our arguments.

Property 15. Any factor of $E$ has degree at most two in each indeterminate $x_{i}$, and joint degree at most three in each pair of indeterminates $x_{i}$ and $x_{j}$.

Thus, for example, $E$ cannot have any term divisible by $x_{1}^{2} x_{2}^{2}$. As another example, if $E=F G$ and $F$ has degree two in some $x_{i}$, then $G$ cannot involve $x_{i}$.

6.1. Linear factors of $E$. We first prove the conclusions of Theorem 6 under the additional assumption that $E$ has a non-monomial linear factor.

Lemma 16. Under the hypotheses of Theorem 6, any non-monomial linear factor of $E$ is a linear combination of exactly two indeterminates.

Proof. Let $E=F G$, with $G$ a non-monomial linear factor. Because $F$ is homogeneous of degree $n-1$ in $n$ indeterminates, according to Property 15 each term of $F$ misses at most two indeterminates.

Now suppose for a contradiction that $G$ involves at least three indeterminates. Then an arbitrary monomial of $F$ must share at least one indeterminate with $G$, say $x_{i}$. But then $F$ has degree exactly one in that $x_{i}$, and joint degree at most two in $x_{i}$ and $x_{j}$, for each other indeterminate $x_{j}$. Consequently, that arbitrary term of $F$ has degree at most one in each indeterminate, and hence $F$ is multilinear.

Writing $F=x_{1} \cdots x_{n} \cdot \sum_{i=1}^{n} f_{i} / x_{i}$ and $G=\sum_{j=1}^{n} g_{j} x_{j}$ and comparing coefficients of the non-multilinear terms of $E$ on both sides of the equation $F G=E$ we find $f_{i} g_{j}=a_{i} b_{j}$ for $i \neq j$. Now our assumption that at least three of the coefficients $g_{j}$ are nonzero implies that the $n$-tuples $\left(f_{1}, \ldots, f_{n}\right)$ and $\left(a_{1}, \ldots, a_{n}\right)$ are proportional. In fact, our equations imply $\left(f_{i} a_{j}-f_{j} a_{i}\right) g_{k} b_{k}=0$ for any distinct $i, j, k$. If $g_{k} \neq 0$ for some $k$, then $b_{k} \neq 0$, otherwise $f_{i}=a_{i} b_{k} / g_{k}=0$ and $f_{j}=a_{j} b_{k} / g_{k}=0$, which is impossible. Hence if $g_{k} \neq 0$ for some $k$, then $f_{i} a_{j}=f_{j} a_{i}$ for any $i, j \neq k$. Consequently, if $g_{k} \neq 0$ for at least three values of $k$, then then $f_{i} a_{j}=f_{j} a_{i}$ for any $i, j$, and hence the $n$-tuples $\left(f_{1}, \ldots, f_{n}\right)$ and $\left(a_{1}, \ldots, a_{n}\right)$ are proportional.

We conclude that $F$ is a scalar multiple of $x_{1} \cdots x_{n} \cdot \sum_{i=1}^{n} a_{i} / x_{i}$, in plain contradiction with the definition of $E$ and the fact that $F G=E$. 
Lemma 17. Under the hypotheses of Theorem 6, if $E$ has $x_{1}+x_{2}$ as a factor, then either

$$
\begin{array}{r}
E /\left(x_{3} \cdots x_{n}\right)=x_{1} x_{2} \cdot\left(1+\left(a / x_{1}+(a+c) / x_{2}\right) \cdot\left(b x_{1}+\left(b-c^{-1}\right) x_{2}\right)\right) \\
=\left(x_{1}+x_{2}\right)\left((a+c) b x_{1}+a\left(b-c^{-1}\right) x_{2}\right)
\end{array}
$$

for some $a, b, c \in \overline{\mathbb{F}_{q}}$ with $c \neq 0$, or

$$
\begin{array}{r}
E /\left(x_{4} \cdots x_{n}\right)=x_{1} x_{2} x_{3} \cdot\left(1+\left(a / x_{1}+a / x_{2}-1 / x_{3}\right) \cdot\left(b x_{1}+b x_{2}-x_{3}\right)\right) \\
=\left(x_{1}+x_{2}\right)\left(a b x_{2} x_{3}+a b x_{1} x_{3}+b x_{1} x_{2}-a x_{3}^{2}\right),
\end{array}
$$

up to permuting the indeterminates $x_{3}, \ldots, x_{n}$.

Proof. Any non-multilinear factor of $F$ cannot involve either $x_{1}$ or $x_{2}$, and hence

$$
F=x_{1} \cdots x_{n} \cdot \sum_{i=1}^{n} f_{i} / x_{i}+x_{3} \cdots x_{n} \cdot \sum_{j=3}^{n} f_{j}^{\prime} x_{j} .
$$

Then the product $E=F G$ has no term of the form $x_{1} \cdots x_{n} \cdot x_{j} / x_{i}$, and so we have $a_{i} b_{j}=0$ whenever $i, j>2$ and $i \neq j$. This implies $a_{i}=b_{i}=0$ for all indices $i>2$ except possibly one, and possibly after permuting the indeterminates $x_{3}, \ldots, x_{n}$ we may assume that $a_{i}=b_{i}=0$ for $i>3$. Therefore, each of the indeterminates $x_{4}, \ldots, x_{n}$ appears in each monomial of $E$ with exponent exactly one, and hence $f_{i}=f_{i}^{\prime}=0$ for $i>3$.

Thus we have $F=\left(f_{1} x_{2} x_{3}+f_{2} x_{1} x_{3}+f_{3} x_{1} x_{2}+f_{3}^{\prime} x_{3}^{2}\right) \cdot x_{4} \cdots x_{n}$, and comparing coefficients of each term on both sides of the equation $F G=E$ we find

$$
\begin{aligned}
f_{2} & =a_{2} b_{1}, \quad f_{3}=a_{3} b_{1}, \quad f_{1}=a_{1} b_{2}, \quad f_{3}=a_{3} b_{2}, \\
f_{1}+f_{2} & =1+a_{1} b_{1}+a_{2} b_{2}+a_{3} b_{3}, \quad f_{3}^{\prime}=a_{2} b_{3}, \quad f_{3}^{\prime}=a_{1} b_{3} .
\end{aligned}
$$

Substituting the first and third equation of the set into the fifth one turns that into $\left(a_{1}-a_{2}\right)\left(b_{1}-b_{2}\right)+a_{3} b_{3}+1=0$. Hence if $a_{3} b_{3}=0$ then $a_{1} \neq a_{2}$ and $b_{1} \neq b_{2}$, whence $f_{3}=f_{3}^{\prime}=0$, and so

$$
F=\left((a+c) b x_{1}+a\left(b-c^{-1}\right) x_{2}\right) \cdot x_{3} \cdots x_{n},
$$

where we have set $a:=a_{1}, b:=b_{1}$, and $c:=a_{2}-a_{1}=\left(b_{1}-b_{2}\right)^{-1}$. However, if $a_{3} b_{3} \neq 0$, then the displayed equations yield $a_{1}=a_{2}, b_{1}=b_{2}$, and $b_{3}=-1 / a_{3}$, whence $f_{1}=f_{2}=a_{1} b_{1}, f_{3}=a_{3} b_{1}, f_{3}^{\prime}=-a_{1} / a_{3}$, that is,

$$
F=\left(a b x_{2} x_{3}+a b x_{1} x_{3}+b x_{1} x_{2}-a x_{3}^{2}\right) \cdot x_{4} \cdots x_{n}
$$

after setting $a:=a_{1} / a_{3}$ and $b:=a_{3} b_{1}$.

6.2. General plan of the proof. Because of Lemmas 16 and 17, in order to prove Theorem 6 it remains to show that if $E=F G$ is any factorisation into non-monomial factors, then either $F$ or $G$ is the product of a monomial and a linear factor. This will be our goal from now on. Hence we may set the following assumptions, which will make the subsequent arguments run smoother. 
Assumptions 18. Let the polynomial $E$ of Theorem 6 be the product of two polynomials $F$ and $G$, of degrees $r>1$ and $n-r>1$, neither of which is a monomial. Assume also that $G$ has no non-trivial monomial factor.

Our assumptions on the degrees are allowed because otherwise either $F$ or $G$ would be the desired non-monomial linear factor. That $G$ has no non-trivial monomial factor can always be achieved by moving any monomial factor from $G$ to $F$.

6.3. The case where either $F$ or $G$ is not multilinear. If $F$ is multilinear but $G$ is not, then we may interchange the roles of $F$ and $G$, after moving any monomial factor so that Assumptions 18 remain satisfied. Hence assume that $F$ is not multilinear.

Possibly after renumbering the indeterminates, we may assume that $F$ has a term $x_{1}^{2} x_{2} \cdots x_{r-1}$. Then $G$ is a multilinear polynomial of degree $n-r$ in the remaining $n-r+1$ indeterminates $x_{r}, \ldots, x_{n}$, otherwise Property 15 would be contradicted. Because $G$ has no non-trivial monomial factor according to Assumptions 18, each term $x_{r} \cdots x_{n} / x_{i}$ with $i \geq r$ appears in $G$ with a nonzero coefficient. In turn, Property 15 implies that any non-multilinear term of $F$ can only involve the indeterminates $x_{1}, \ldots, x_{r-1}$.

If some (multilinear) term of $F$ involved at least two of the indeterminates $x_{r}, \ldots, x_{n}$, then because $n-r>1$ that term would share at least two indeterminates with some term of $G$, and this would contradict Property 15 . Therefore, no term of $F$ involves more than one indeterminate from $x_{r}, \ldots, x_{n}$. We have seen earlier that any term of $F$ which involves such indeterminate must be multilinear, and so altogether $F$ can be written in the form

$$
F=x_{1} \cdots x_{r-1} \cdot \sum_{i=1}^{n} f_{i} x_{i},
$$

which provides us with the desired linear factor.

6.4. The case where $F$ and $G$ are both multilinear. Now we may suppose that both $F$ and $G$ are multilinear polynomials. Because of Property 15 each term of $G$ can share at most one indeterminate with each term of $F$. Any two distinct terms of $F$ must involve together exactly $r+1$ or $r+2$ indeterminates. In fact, if they did involve more, then any term of $G$ would involve at least three of them, and hence it would share at least two indeterminates with at least one of the two terms of $F$ under consideration, contradicting Property 15 . We deal with those two cases separately.

6.5. The subcase $r+1$. Suppose first that $F$ has at least two terms which together involve $r+1$ indeterminates. After renumbering the indeterminates we may assume that two such terms are $f_{r} x_{1} \cdots x_{r}+f_{r+1} x_{1} \cdots x_{r-1} \cdot x_{r+1}$, with $f_{r}, f_{r+1} \neq 0$. Because of Property 15 each term of $G$ can share at most one 
indeterminate with each of them, and hence

$$
G=\sum_{i=1}^{r+1} g_{i} x_{i} \cdot x_{r+2} \cdots x_{n}+\sum_{j=r+2}^{n} g_{j}^{\prime} x_{r} \cdots x_{n} / x_{j}
$$

for some scalars $g_{i}, g_{i}^{\prime}$. An alternative choice of notation would be restricting the former summation range to $i<r$ and extending the latter to $i \geq r$, provided we set $g_{r}^{\prime}=g_{r+1}$ and $g_{r+1}^{\prime}=g_{r}$. We conveniently allow this double notation in what follows.

Comparing the coefficients of corresponding monomials on both sides of the equality $F G=E$ we find, in particular,

$$
\begin{gathered}
f_{r} g_{i}=a_{r+1} b_{i} \quad \text { and } \quad f_{r+1} g_{i}=a_{r} b_{i} \quad \text { for } i<r \\
f_{r} g_{j}^{\prime}=a_{j} b_{r} \quad \text { and } \quad f_{r+1} g_{j}^{\prime}=a_{j} b_{r+1} \quad \text { for } j>r+1 .
\end{gathered}
$$

In fact, because $F$ is multilinear any term in the product $F G$ where $x_{i}$ appears with exponent two, for some $i<r$, can only arise in one way, as the product of the term of $G$ with coefficient $g_{i}$ and a uniquely determined term of $F$, necessarily one of the two considered above. A similar argument applies to any term in the product $F G$ which misses the indeterminate $x_{j}$, where $j>r+1$. Because $F$ is multilinear, Equation (6.1) implies that $E=F G$ has no term of the form $x_{1} \cdots x_{n} \cdot x_{i} / x_{j}$ with $i<r$ and $j>r+1$, which means $a_{j} b_{i}=0$. Hence either $b_{i}=0$ for all $i<r$, or $a_{j}=0$ for all $j>r+1$. However, the latter together with Equation (6.2) yields that $g_{j}^{\prime}=0$ for $j>r+1$, whence $G=x_{r+2} \cdots x_{n} \cdot \sum_{i=1}^{r+1} g_{i} x_{i}$ has a non-trivial monomial factor, against Assumptions 18. We conclude that $b_{i}=0$ for $i<r$, and Equation (6.2) yields $g_{i}=0$ for $i<r$, and so $G=$ $\sum_{j=r}^{n} g_{j}^{\prime} x_{r} \cdots x_{n} / x_{j}$.

Because $G$ has no non-trivial monomial factor we have $g_{j}^{\prime} \neq 0$ for $j \geq r$. This implies that no term of $F$ can involve more than one indeterminate from the set $\left\{x_{r}, \ldots, x_{n}\right\}$, and hence

$$
F=x_{1} \cdots x_{r-1} \cdot \sum_{j=r}^{n} f_{j} x_{j}
$$

providing us with the desired linear factor.

6.6. The subcase $r+2$. Now we may assume that each two distinct terms of $F$ involve together exactly $r+2$ indeterminates. We will deduce a contradiction. Possibly after renumbering the indeterminates we may assume that two of the terms of $F$ are (nonzero) scalar multiples of $x_{1} x_{2} \cdot x_{5} \cdots x_{r+2}$ and $x_{3} x_{4} \cdot x_{5} \cdots x_{r+2}$ (to be appropriately interpreted in case $r=2$ ). Because each term of $G$ involves exactly $n-r$ indeterminates, and can share at most one indeterminate with each of those two terms of $F$ according to Property 15, it must involve all of $x_{r+3}, \ldots, x_{n}$, and exactly one indeterminate from each of the two sets $\left\{x_{1}, x_{2}\right\}$ and $\left\{x_{3}, x_{4}\right\}$. Because $G$ has no non-trivial monomial factor we have $r=n-2$, and hence

$$
G=g_{13} x_{1} x_{3}+g_{14} x_{1} x_{4}+g_{23} x_{2} x_{3}+g_{24} x_{2} x_{4}
$$


for certain scalars $g_{i j}$. Again because $G$ has no non-trivial monomial factor we have either $g_{13} g_{24} \neq 0$ or $g_{14} g_{23} \neq 0$. After possibly exchanging $x_{3}$ and $x_{4}$ we may assume the former.

Now according to Property 15 each term of $F$ can share at most one indeterminate with each term of $G$, and this leaves only $x_{1} x_{4} \cdot x_{5} \cdots x_{n}$ and $x_{2} x_{3} \cdot x_{5} \cdots x_{n}$ as possible terms of $F$ besides those two assumed from the start. However, each of these must be excluded because together with one of the initial terms it involves only $n-1=r+1$ indeterminates, rather than $r+2$. Hence we have

$$
F=\left(f_{12} x_{1} x_{2}+f_{34} x_{3} x_{4}\right) \cdot x_{5} \cdots x_{n}
$$

with $f_{12} f_{34} \neq 0$. Comparing coefficients of corresponding terms in the equality $F G=E$ we find $f_{12} g_{13}=a_{4} b_{1}$ and $f_{34} g_{13}=a_{2} b_{3}$, whence $a_{2} b_{1} \neq 0$. This means that $F G$ has a nonzero term $x_{1}^{2} x_{3} x_{4} \cdot x_{5} \cdots x_{n}$, which is clearly impossible.

This contradiction completes our proof of Theorem 6 .

\section{REFERENCES}

[CDVS09] Andrea Caranti, Francesca Dalla Volta, and Massimiliano Sala, An application of the O'Nan-Scott theorem to the group generated by the round functions of an AES-like cipher, Des. Codes Cryptogr. 52 (2009), no. 3, 293-301. MR 2506729 (2010a:94053)

[Csa13] Bence Csajbók, Linear subspaces of finite fields with large inverse-closed subsets, Finite Fields Appl. 19 (2013), 55-66. MR 2996759

[EOT10] Jordan S. Ellenberg, Richard Oberlin, and Terence Tao, The Kakeya set and maximal conjectures for algebraic varieties over finite fields, Mathematika 56 (2010), no. 1, 1-25. MR 2604979 (2011c:14066)

[GGSZ06] Daniel Goldstein, Robert M. Guralnick, Lance Small, and Efim Zelmanov, Inversion invariant additive subgroups of division rings, Pacific J. Math. 227 (2006), no. 2, 287-294. MR 2263018 (2007i:17041)

[Hua49] Loo-Keng Hua, Some properties of a sfield, Proc. Nat. Acad. Sci. U. S. A. 35 (1949), 533-537. MR 0031471 (11,155c)

[KLS12] Gábor Korchmáros, Valentino Lanzone, and Angelo Sonnino, Projective k-arcs and 2-level secret-sharing schemes, Des. Codes Cryptogr. 64 (2012), no. 1-2, 3-15. MR 2914398

[LN83] Rudolf Lidl and Harald Niederreiter, Finite fields, Encyclopedia of Mathematics and its Applications, vol. 20, Addison-Wesley Publishing Company Advanced Book Program, Reading, MA, 1983, With a foreword by P. M. Cohn. MR 746963 (86c:11106)

[LW54] Serge Lang and André Weil, Number of points of varieties in finite fields, Amer. J. Math. 76 (1954), 819-827. MR 0065218 (16,398d)

[Mat] Sandro Mattarei, A property of the inverse of a subspace of a finite field, arXiv:1312.1293, to appear in Finite Fields Appl.

[Mat07] _ Inverse-closed additive subgroups of fields, Israel J. Math. 159 (2007), 343347. MR 2342485 (2008j:12008)

E-mail address: mattarei@science.unitn.it

Dipartimento di Matematica, Università degli Studi di Trento, via Sommarive 14, I-38123 Povo (Trento), Italy 